\title{
Multi-priority Cartesian Impedance Control
}

\author{
Robert Platt Jr. \\ Computer Science and Artificial Intelligence Laboratory \\ Massachusetts Institute of Technology \\ rplatt@csail.mit.edu
}

\author{
Muhammad Abdallah, Charles Wampler \\ Manufacturing Systems Research Lab \\ General Motors \\ \{muhammad.abdallah,charles.w.wampler\}@gm.com
}

\begin{abstract}
Manipulator compliance is well known to be important to robot manipulation and assembly. Recently, this has been highlighted by the development of new higly-compliant robot manipulators such as the Barrett arm or the DLR lightweight manipulator [1, 2]. It is also clear that dexterous manipulation involves touching the environment at different locations simultaneously (perhaps at different points on the robot hand or fingers). In these situations, it is particularly attractive to control the system using a multi-priority strategy where several contact points are commanded in parallel. Multi-priority Cartesian impedance control is the natural combination of these two ideas. The system realizes several impedances with different reference positions at different points on the robot with a specified order of priority. We find a controller that minimizes an arbitrary quadratic norm on the second-priority impedance error subject to constraints deriving from the first priority impedance task. We also show that the locally optimal controller does not require force feedback in its implementation for passive desired inertias. The results are illustrated in simulation.
\end{abstract}

\section{INTRODUCTION ${ }^{1}$}

Since the earliest days of robotics, researchers have been aware of the possibility of controlling second-priority objectives using degrees of freedom (DOFs) that are redundant with respect to a first-priority task [3, 4, 5]. However, the advent of high-DOF humanoid robots make it possible to consider executing more than two tasks simultaneously [6, 7]. This approach to robot control is particularly relevant to manipulation tasks where high-DOF hand-arm systems must be controlled so as to interact with an environment at a few desired contact regions. However, it is ironic that although end-effector compliance is important to robust manipulation, relatively little research exists that studies multipriority impedance control. This paper addresses this deficit by proposing a locally-optimal dual-priority impedance law. First, we propose a general law that uses force feedback to realize an arbitrary dual-priority impedance. Then, we characterize the subset of impedances that can be realized using torque controlled manipulators without force sensors. In contrast to prior work, we consider the space of all possible control laws that realize the first-priority impedance and select the one that is optimal with respect to an arbitrary quadratic optimization criterion.

The multi-priority approach to robot manipulator control has been studied extensively. Many early approaches calculated

\footnotetext{
${ }^{1}$ Portions of this publication have patents pending.
}

joint velocities that attempt to achieve a second-priority objective (such as avoiding obstacles or manipulator singularities) while achieving a desired end-effector velocity [4, 5, 8]. Chiaverini considers a damped-least-squares version of the control law that is robust to algorithmic singularities [9]. Antonelli provides a Lyapunov analysis demonstrating that the basic approach is stable [10]. Related approaches have been applied in more general contexts [11, 12].

A significant body of work explores the general problem of impedance control in the context of redundant manipulators. Building on Hogan's early work [13], Natale et. al. propose a version of the impedance controller that correctly handles angular impedances [14]. Albu-Schaffer and Hirzinger propose a dual-priority impedance architecture where the end-effector impedance and null space joint impedance are controlled separately using a stiffness formulation [15] and $\mathrm{Oh}$ et. al. propose an impedance control formulation where task and redundant space tasks are dynamically decoupled [16]. Ott et. al. demonstrate stability even though the redundant space is non-integrable [17].

In contrast to the above, the current paper considers the question of simultaneously controlling the impedance at multiple end-effectors. Rather than stabilizing an arbitrary parameterization of the redundant space, this paper optimizes for an integrable operational space objective. To our knowledge, the most closely related work on the subject is the multipriority framework of Sentis and Khatib [6, 18]. In their work, the multi-priority objectives are realized by projecting lower-priority objectives into the dynamically consistent null space. Although this formulation keeps the first priority task independent of the lower-priority tasks, it does not optimize for second-priority performance. The current paper explores this point. We provide a control law that minimizes quadratic error with respect to the desired lower-priority impedance while still ensuring independence of the first-priority objective. It turns out that for the optimal dual-priority controller, it is possible to identify the operational space directions where the second-priority task is independent of the first-priority task. These independent directions can be adjusted by changing the weighting matrix used by the optimization criterion.

\section{BACKGROUND}

\section{A. Generalized equation of motion}

The dynamic motion of a robot arm with $n$ revolute joints is typically understood in terms of the following generalized 
equation of motion:

$$
M \ddot{q}+\eta=\tau_{a}+\tau,
$$

where $M$ is the $n \times n$ manipulator inertia matrix, $\ddot{q}$ is an $n$-vector of manipulator joint accelerations, $\tau$ is an $n$-vector of joint torques resulting from externally applied loads, $\tau_{a}$ is a vector of actuator torques, and $\eta$ describes the sum of frictional, coriolis, centrifugal, and gravitational torques [19]. The dependence of these terms on manipulator configuration is implicitly assumed. In order to simplify notation, we introduce the following substitution:

$$
\tau_{a}=u+\eta,
$$

such that the equation of motion can be expressed in terms of a command vector, $u$ :

$$
M \ddot{q}=u+\tau .
$$

\section{B. Operational space}

It is frequently useful to design controllers defined in operational space coordinates rather than in joint space. While the term "operational space" may refer to any coordinate system relevant to a robot task, it usually refers to the space of positions and orientations of the end-effector represented by a parameterization of $S E(3)$. In this paper, $S E(3)$ is parameterized using exponential coordinates whereby a Cartesian pose is encoded by a 6-vector with the first three numbers describing position and the last three numbers describing orientation using the axis-angle representation [20]. The Cartesian velocity of the end-effector will be represented as a twist and the acceleration as the derivative of twist. Similarly, loads in Cartesian space will be written as wrenches (six-vector that concatenates a force and a moment) [20]. The end-effector Jacobian, $J$, relates joint velocities, $\dot{q}$, to Cartesian twists at the end-effector, $\dot{x}: \dot{x}=J \dot{q}$.

\section{DUAL PRIORITY CARTESIAN SPACE IMPEDANCE CONTROL}

Dual priority Cartesian impedance control is defined with respect to two points of reference (PORs). A POR is a reference frame attached to the manipulator that is the object of Cartesian control. In general, dual priority Cartesian impedance control realizes two different impedance laws defined with respect to two different PORs simultaneously. One impedance objective is first-priority while the other is secondpriority. Dual-priority Cartesian impedance control minimizes error with respect to the first-priority impedance objective while also realizing the second-priority objective to the greatest extent possible.

\section{A. Problem statement}

Let $x_{1}, \dot{x}_{1}, \ddot{x}_{1}$, and $f_{1}$ be the pose, twist, acceleration, and externally applied wrench associated with the first-priority POR. Define $x_{2}, \dot{x}_{2}, \ddot{x}_{2}$, and $f_{2}$ similarly for the secondpriority POR. The goal of dual-priority impedance control is to realize the first-priority impedance objective,

$$
\Omega_{1} \ddot{x}_{1}+f_{1}^{*}=f_{1},
$$

as completely as possible given the mechanics of the manipulator, where

$$
f_{1}^{*}=B_{1} \dot{x}_{1}+K_{1} \tilde{x}_{1},
$$

$\Omega_{1}$ is the desired inertia, $B_{1}$ is the desired damping, $K_{1}$ is the desired stiffness, and $\tilde{x}_{1}$ is the pose error in the first-priority POR. In addition, dual-priority Cartesian impedance control must also minimize error with respect to the second-priority impedance objective,

$$
\Omega_{2} \ddot{x}_{2}+f_{2}^{*}=f_{2},
$$

to the greatest extent possible while not impacting performance with regard to the first-priority objective, where

$$
f_{2}^{*}=B_{2} \dot{x}_{2}+K_{2} \tilde{x}_{2},
$$

$\Omega_{2}$ is the desired inertia, $B_{2}$ is the desired damping, $K_{2}$ is the desired stiffness, and $\tilde{x}_{2}$ is the pose error at the second-priority POR.

\section{B. Optimization criterion}

The possibility of realizing two Cartesian space impedances depends on the degree of mobility between the two PORs. In general, it is not possible to realize both Cartesian impedances concurrently because the two PORs are not necessarily sufficiently mobile with respect to each other. Therefore, the multiple-priority approach applies actuator torques such that the primary impedance objective is achieved while minimizing an optimization criterion associated with the second-priority impedance objective. We restrict our attention to the case where the optimization criterion is the weighted squared magnitude of the Cartesian acceleration at the second-priority POR:

$$
\begin{aligned}
\epsilon & =\left(\ddot{x}_{2}-\ddot{x}_{2}^{\prime}\right)^{T} W\left(\ddot{x}_{2}-\ddot{x}_{2}^{\prime}\right) \\
& =z^{T} z,
\end{aligned}
$$

where $\ddot{x}_{2}^{\prime}$ is the desired Cartesian space acceleration at the second-priority POR and:

$$
z=W^{\frac{1}{2}}\left(\ddot{x}_{2}-\ddot{x}_{2}^{\prime}\right)
$$

The goal of dual-priority Cartesian impedance control is to minimize Equation 6 while realizing the first-priority impedance in Equation 2.

\section{Control law}

The first-priority Cartesian impedance constrains the space of joint accelerations that must be considered. Substituting $J_{1} \ddot{q}+\dot{J}_{1} \dot{q}$ for $\ddot{x}_{1}$ in Equation 2 and taking the pseudo-inverse, we have:

$$
\ddot{q}=J_{1}^{+}\left[\Omega_{1}^{-1}\left(f_{1}-f_{1}^{*}\right)-\dot{J} \dot{q}\right]+N_{1} \lambda,
$$

where $N_{1}=I-J_{1}^{+} J_{1}$ and $\lambda$ is arbitrary. Using $\ddot{x}_{2}=J_{2} \ddot{q}+$ $\dot{J}_{2} \dot{q}$, the space of Cartesian accelerations at the second-priority POR consistent with the first-priority impedance is:

$$
\ddot{x}_{2}=J_{2} J_{1}^{+}\left[\Omega_{1}^{-1}\left(f_{1}-f_{1}^{*}\right)-\dot{J} \dot{q}\right]+\dot{J}_{2} \dot{q}+J_{2} N_{1} \lambda .
$$


Among those joint accelerations permitted by the constraint, we must find one that minimizes the optimization criterion. This can be achieved by solving Equation 9 for the value of $\lambda$ that minimizes $\epsilon$ (Equation 6), or equivalently, $z$ (Equation 7). Solving Equation 4 for the desired acceleration at the second POR, we have:

$$
\ddot{x}_{2}^{\prime}=\Omega_{2}^{-1}\left(f_{2}-f_{2}^{*}\right) .
$$

Substituting Equation 9 into Equation 7 for $\ddot{x}_{2}$ and using $\ddot{x}_{2}^{\prime}$ above, we have:

$$
\begin{aligned}
z= & W^{\frac{1}{2}}\left(\ddot{x}_{2}-\ddot{x}_{2}^{\prime}\right) \\
= & W^{\frac{1}{2}}\left[J_{2} J_{1}^{+}\left(\Omega_{1}^{-1}\left(f_{1}-f_{1}^{*}\right)-\dot{J}_{1} \dot{q}\right)+\dot{J}_{2} \dot{q}\right. \\
& \left.-\Omega_{2}^{-1}\left(f_{2}-f_{2}^{*}\right)+J_{2} N_{1} \lambda\right] .
\end{aligned}
$$

The value of $\lambda$ that minimizes $z^{T} z$ can be found using the pseudo-inverse:

$$
\begin{aligned}
\lambda= & \hat{J}_{2}\left[\Omega_{2}^{-1}\left(f_{2}-f_{2}^{*}\right)-\dot{J}_{2} \dot{q}\right] \\
& -\hat{J}_{2} J_{2} J_{1}^{+}\left[\Omega_{1}^{-1}\left(f_{1}-f_{1}^{*}\right)-\dot{J}_{1} \dot{q}\right] \\
& +\left(I-\hat{J}_{2} J_{2} N_{1}\right) \beta
\end{aligned}
$$

where

$$
\hat{J}_{2}=\left(W^{\frac{1}{2}} J_{2} N_{1}\right)^{+} W^{\frac{1}{2}} .
$$

The term $\left(I-\hat{J}_{2} J_{2} N_{1}\right) \beta$ (for arbitrary $\beta$ ) represents the additional freedoms left over in the case that the secondpriority optimization criterion does not completely determine the motion of all joints. The matrix $\left(I-\hat{J}_{2} J_{2} N_{1}\right)$ is an orthogonal projection matrix that spans the joint accelerations irrelevant to either objective.

The joint accelerations that realize the first-priority impedance (Equation 2) while also minimizing the optimization criterion with respect to the second-priority impedance (Equation 4) can be found by substituting $\lambda$ back into Equation 8:

$$
\begin{aligned}
\ddot{q}= & J_{1}^{+}\left[\Omega_{1}^{-1}\left(f_{1}-f_{1}^{*}\right)-\dot{J}_{1} \dot{q}\right] \\
& +N_{1} \hat{J}_{2}\left[\Omega_{2}^{-1}\left(f_{2}-f_{2}^{*}\right)-\dot{J}_{2} \dot{q}\right] \\
& -N_{1} \hat{J}_{2} J_{2} J_{1}^{+}\left[\Omega_{1}^{-1}\left(f_{1}-f_{1}^{*}\right)-\dot{J}_{1} \dot{q}\right] \\
& +N_{1}\left(I-\hat{J}_{2} J_{2} N_{1}\right) \beta .
\end{aligned}
$$

This equation can be simplified by using the fact that for any matrix, $A$, the following is true: $A^{+}=A^{T}\left(A A^{T}\right)^{+}$. Therefore, we have that:

$$
\begin{aligned}
N_{1} \hat{J}_{2} & =N_{1}\left(W^{\frac{1}{2}} J_{2} N_{1}\right)^{+} W^{\frac{1}{2}} \\
& =N_{1}\left(N_{1} J_{2}^{T} W^{\frac{1}{2}}\right)\left(W^{\frac{1}{2}} J_{2} N_{1} J_{2}^{T} W^{\frac{1}{2}}\right)^{+} W^{\frac{1}{2}} \\
& =\left(N_{1} J_{2}^{T} W^{\frac{1}{2}}\right)\left(W^{\frac{1}{2}} J_{2} N_{1} J_{2}^{T} W^{\frac{1}{2}}\right)^{+} W^{\frac{1}{2}} \\
& =\hat{J}_{2} .
\end{aligned}
$$

As a result, Equation 13 becomes:

$$
\begin{aligned}
\ddot{q}= & \hat{N}_{2} J_{1}^{+}\left[\Omega_{1}^{-1}\left(f_{1}-f_{1}^{*}\right)-\dot{J}_{1} \dot{q}\right] \\
& +\hat{J}_{2}\left[\Omega_{2}^{-1}\left(f_{2}-f_{2}^{*}\right)-\dot{J}_{2} \dot{q}\right] \\
& +N_{1} \hat{N}_{2} N_{1} \beta
\end{aligned}
$$

where

$$
\hat{N}_{2}=\left(I-\hat{J}_{2} J_{2}\right) .
$$

Substituting into the equation of motion (Equation 1) for $\ddot{q}$, we have:

$$
\begin{aligned}
u= & M \ddot{q}-\tau \\
= & M \hat{N}_{2} J_{1}^{+}\left[\Omega_{1}^{-1}\left(f_{1}-f_{1}^{*}\right)-\dot{J}_{1} \dot{q}\right] \\
& +M \hat{J}_{2}\left[\Omega_{2}^{-1}\left(f_{2}-f_{2}^{*}\right)-\dot{J}_{2} \dot{q}\right] \\
& +M N_{1} \hat{N}_{2} N_{1} \beta-\tau .
\end{aligned}
$$

In order to realize Equation 17 in practice, it is necessary to measure the externally applied loads. This might be accomplished by measuring the loads, $f_{1}$ and $f_{2}$, applied at the first- and second-priority PORs and assuming that no external loads are applied elsewhere on the manipulator. Accordingly, we have:

$$
\tau=J_{1}^{T} f_{1}+J_{2}^{T} f_{2} .
$$

Substituting into Equation 17, we have:

$$
\begin{aligned}
u= & M \ddot{q}-\tau \\
= & M \hat{N}_{2} J_{1}^{+}\left[\Omega_{1}^{-1}\left(f_{1}-f_{1}^{*}\right)-\dot{J}_{1} \dot{q}\right] \\
& +M \hat{J}_{2}\left[\Omega_{2}^{-1}\left(f_{2}-f_{2}^{*}\right)-\dot{J}_{2} \dot{q}\right] \\
& +M N_{1} \hat{N}_{2} N_{1} \beta-J_{1}^{T} f_{1}-J_{2}^{T} f_{2} .
\end{aligned}
$$

The control law in Equation 18 solves the problem proposed in Section III-A while minimizing the Cartesian acceleration of the second-priority POR and assuming that externally applied wrenches at each POR can be measured directly.

\section{Analysis}

The closed-loop behavior of the dual-priority Cartesian impedance controller (Equation 18) can be understood in terms of the Cartesian accelerations at the first- and second-priority PORs. First, note that:

$$
\begin{aligned}
J_{1} \hat{J}_{2} & =J_{1}\left(W^{\frac{1}{2}} J_{2} N_{1}\right)^{+} W^{\frac{1}{2}} \\
& =J_{1} N_{1} J_{2}^{T} W^{\frac{1}{2}}\left(W^{\frac{1}{2}} J_{2} N_{1} J_{2}^{T} W^{\frac{1}{2}}\right)^{+} W^{\frac{1}{2}} \\
& =0 .
\end{aligned}
$$

Therefore, we have that:

$$
\begin{aligned}
J_{1} \hat{N}_{2} & =J_{1}\left(I-\hat{J}_{2} J_{2}\right) \\
& =J_{1} .
\end{aligned}
$$

Using the above results and substituting Equation 15 into $\ddot{x}_{1}=$ $J_{1} \ddot{q}+\dot{J}_{1} \dot{q}$, the Cartesian acceleration at the first POR is:

$$
\ddot{x}_{1}=\Omega_{1}^{-1}\left(f_{1}-f_{1}^{*}\right),
$$


thereby realizing the desired first-priority impedance.

The Cartesian acceleration of the second-priority POR can be calculated using $\ddot{x}_{2}=J_{2} \ddot{q}+\dot{J}_{2} \dot{q}$ :

$$
\begin{aligned}
\ddot{x}_{2}= & J_{2} \hat{J}_{2}\left[\Omega_{2}^{-1}\left(f_{2}-f_{2}^{*}\right)-\dot{J}_{2} \dot{q}\right]+\dot{J}_{2} \dot{q} \\
& +\left(I-J_{2} \hat{J}_{2}\right) J_{2} J_{1}^{+}\left[\Omega_{1}^{-1}\left(f_{1}-f_{1}^{*}\right)-\dot{J}_{1} \dot{q}\right] \\
& +J_{2} N_{1} \hat{N}_{2} N_{1} \beta .
\end{aligned}
$$

The last term of this equation can be eliminated by noting:

$$
\begin{aligned}
J_{2} N_{1} \hat{N}_{2} N_{1}= & J_{2} N_{1}\left(I-\hat{J}_{2} J_{2}\right) N_{1} \\
= & J_{2} N_{1}-J_{2} N_{1} \hat{J}_{2} J_{2} N_{1} \\
= & J_{2} N_{1}-J_{2} N_{1}\left(W^{\frac{1}{2}} J_{2} N_{1}\right)^{+} W^{\frac{1}{2}} J_{2} N_{1} \\
= & W^{-\frac{1}{2}}\left[W^{\frac{1}{2}} J_{2} N_{1}\right. \\
& \left.-W^{\frac{1}{2}} J_{2} N_{1}\left(W^{\frac{1}{2}} J_{2} N_{1}\right)^{+} W^{\frac{1}{2}} J_{2} N_{1}\right] \\
= & 0 .
\end{aligned}
$$

Therefore, the resulting closed-loop impedance at $x_{2}$ is:

$$
\begin{aligned}
\ddot{x}_{2}= & J_{2} \hat{J}_{2}\left[\Omega_{2}^{-1}\left(f_{2}-f_{2}^{*}\right)-\dot{J_{2}} \dot{q}\right]+\dot{J}_{2} \dot{q} \\
& +\left(I-J_{2} \hat{J}_{2}\right) J_{2} J_{1}^{+}\left[\Omega_{1}^{-1}\left(f_{1}-f_{1}^{*}\right)-\dot{J}_{1} \dot{q}\right]
\end{aligned}
$$

Equation 20 can be understood by recognizing that $J_{2} \hat{J}_{2}$ and $\left(I-J_{2} \hat{J}_{2}\right)$ are orthogonal weighted projection matrices: $J_{2} \hat{J}_{2}\left(I-J_{2} \hat{J}_{2}\right)=0$. The first term is projected through $J_{2} \hat{J}_{2}$ and describes the contribution of the second-priority impedance to the closed-loop acceleration of $x_{2}$. The last term is projected through $\left(I-J_{2} \hat{J}_{2}\right)$ and describes the effect that the first-priority impedance objective has on the closed loop behavior at the second-priority POR. Notice that the secondpriority impedance operates completely independently in the range space of $J_{2} \hat{J}_{2}$. Multiplying both sides of Equation 20 by $J_{2} \hat{J}_{2}$, we have:

$$
J_{2} \hat{J}_{2} \ddot{x}_{2}=J_{2} \hat{J}_{2} \Omega_{2}^{-1}\left(f_{2}-f_{2}^{*}\right) .
$$

\section{E. Comparison to Sentis-Khatib}

These closed-loop dynamics may be compared to the dynamics of the multi-priority Sentis-Khatib control law. Based on [6], the Sentis-Khatib control law is:

$$
\begin{aligned}
\tau= & J_{1}^{T} \Lambda_{1}\left[\Omega_{1}^{-1}\left(f_{1}-f_{1}^{*}\right)-\dot{J}_{1} \dot{q}\right] \\
& +J_{2 \mid 1}^{T} \Lambda_{2 \mid 1}\left[\Omega_{2}^{-1}\left(f_{2}-f_{2}^{*}\right)-\dot{J}_{2} \dot{q}\right] .
\end{aligned}
$$

In the above control law, we have used

$$
\Lambda_{2 \mid 1}=\left(J_{2 \mid 1} M^{-1} J_{2 \mid 1}^{T}\right)^{-1}
$$

and

$$
J_{2 \mid 1}=J_{2} N_{M},
$$

where $N_{A}$ is the inertia-weighted null space of the first priority objective:

$$
N_{M}=I-M^{-1} J_{1}^{T}\left(J_{1} M^{-1} J_{1}^{T}\right)^{-1} J_{1}
$$

Substituting back into the equation of motion (Equation 1) and solving for $\ddot{x}_{1}$, we arrive at Equation 2, demonstrating that the second-priority objective does not influence the dynamics of the first-priority objective. However, substituting into the equation of motion and solving for $\ddot{x}_{2}$, we get:

$$
\begin{aligned}
\ddot{x}_{2}= & J_{2} M^{-1} J_{1}^{T} \Lambda_{1} \Omega_{1}^{-1}\left(f_{1}-f_{1}^{*}\right) \\
& +J_{2} M^{-1} J_{2 \mid 1}^{T} \Lambda_{2 \mid 1} \Omega_{2}^{-1}\left(f_{2}-f_{2}^{*}\right) .
\end{aligned}
$$

It should be noted that the second term above is not orthogonal to the first term as is the case in Equation 20. This begs the question of whether this control law is optimal for any criterion of the form of Equation 6. This question can be answered using the Lagrange multiplier method. We are interested in control laws that minimize

$$
f(\ddot{q})=\left(J_{2} \ddot{q}-\beta\right)^{T} W\left(J_{2} \ddot{q}-\beta\right)
$$

subject to

$$
g(\ddot{q})=J_{1} \ddot{q}-\alpha=0,
$$

where $\alpha=\Omega_{1}^{-1}\left(f_{1}-f_{1}^{*}\right)-\dot{J}_{1} \dot{q}$ and $\beta=\Omega_{2}^{-1}\left(f_{2}-f_{2}^{*}\right)-\dot{J}_{2} \dot{q}$. Differentiating $f$ and $g$ and constructing the Lagrangian, we have

$$
h^{T} J_{1}+\ddot{q}^{T} J_{2}^{T} W J_{2}-\beta^{T} W J_{2}=0,
$$

where $h$ is the Lagrange multiplier. Equation 23 must be satisfied for some value of $h$ at an optimum. Equivalently, we can require:

$$
N_{1}\left[J_{2}^{T} W J_{2} \ddot{q}-J_{2}^{T} W \beta\right]=0 .
$$

Solving for $\ddot{q}$ by substituting Equation 22 into Equation 1 and substituting into the above, the condition becomes:

$$
N_{1}\left[J_{2}^{T} W J_{2} M^{-1}\left(J_{1}^{T} \Lambda_{1} \alpha+J_{2 \mid 1}^{T} \Lambda_{2 \mid 1} \beta\right)-J_{2}^{T} W \beta\right]=0 .
$$

Since we must assume $\alpha$ to be arbitrary, the condition is only satisfied when

$$
N_{1} J_{2}^{T} W J_{2} M^{-1} J_{1}^{T} \Lambda_{1} \alpha=0 .
$$

However, since there is no fixed $W$ such that Equation 24 is true for arbitrary $\alpha$, Equation 22 cannot be optimal.

\section{IMPEDANCE CONTROL WITHOUT FORCE SENSORS}

An important concern with multi-priority impedance control as it is expressed in Equation 18 is the need to sense externally applied loads at two different points on the manipulator. While this may be feasible in some cases, it is important to address the case where such feedback is not available.

\section{A. Control law}

Consider Equation 17, and suppose that the joint torque caused by all externally applied loads $(\tau)$ is substituted for each instance of $J_{1}^{T} f_{1}$ or $J_{2}^{T} f_{2}$. Let $\Lambda_{1}=\left(J_{1} M^{-1} J_{1}^{T}\right)^{-1}$ be the passive manipulator inertia described in Cartesian coordinates at the first-priority POR. Let $\Lambda_{2}=\left(J_{2} M^{-1} J_{2}^{T}\right)^{-1}$ be the similar quantity at the second-priority POR. Set the 
desired Cartesian inertias to these passive values: $\Omega_{1}=\Lambda_{1}$ and $\Omega_{2}=\Lambda_{2}$. Then, Equation 17 becomes:

$$
\begin{aligned}
u= & -M \hat{N}_{2} J_{1}^{+}\left[\Lambda_{1}^{-1} f_{1}^{*}+\dot{J}_{1} \dot{q}\right] \\
& -M \hat{J}_{2}\left[\Lambda_{2}^{-1} f_{2}^{*}+\dot{J}_{2} \dot{q}\right] \\
& +M \hat{N}_{2} J_{1}^{+} J_{1} M^{-1} \tau \\
& +M \hat{J}_{2} J_{2} M^{-1} \tau \\
& +M N_{1} \hat{N}_{2} N_{1} \beta \\
& -\tau .
\end{aligned}
$$

Since the value of $\beta$ does not affect the closed-loop behavior of the control law with respect to the first- or second-priority impedance objectives, set $\beta=M^{-1} \tau+\gamma$, where $\gamma$ is arbitrary. Then the last four terms of Equation 25 are:

$$
M\left[\hat{N}_{2} J_{1}^{+} J_{1}+\hat{J}_{2} J_{2}+N_{1} \hat{N}_{2} N_{1}\right] M^{-1} \tau-\tau+N_{1} \hat{N}_{2} N_{1} \gamma
$$

This expression can be simplified using Equation 14 to find that

$$
\begin{aligned}
N_{1} \hat{N}_{2} N_{1} & =N_{1}\left(I-\hat{J}_{2} J_{2}\right) N_{1} \\
& =\left(N_{1}-N_{1} \hat{J}_{2} J_{2}\right) N_{1} \\
& =\left(N_{1}-\hat{J}_{2} J_{2}\right) N_{1} \\
& =\left(I-\hat{J}_{2} J_{2}\right) N_{1} \\
& =\hat{N}_{2} N_{1} .
\end{aligned}
$$

Therefore, the last four terms of Equation 25 can be simplified as follows:

$$
\begin{aligned}
& M\left[\hat{N}_{2} J_{1}^{+} J_{1}+\hat{J}_{2} J_{2}+\hat{N}_{2} N_{1}\right] M^{-1} \tau-\tau+\hat{N}_{2} N_{1} \gamma \\
= & M\left[\hat{N}_{2}+\hat{J}_{2} J_{2}\right] M^{-1} \tau-\tau+\hat{N}_{2} N_{1} \gamma \\
= & M M^{-1} \tau-\tau+\hat{N}_{2} N_{1} \gamma \\
= & \hat{N}_{2} N_{1} \gamma .
\end{aligned}
$$

As a result, Equation 25 can be re-written without the $\tau$ terms:

$$
\begin{aligned}
u= & -M \hat{N}_{2} J_{1}^{+}\left[\Lambda_{1}^{-1} f_{1}^{*}+\dot{J}_{1} \dot{q}\right] \\
& -M \hat{J}_{2}\left[\Lambda_{2}^{-1} f_{2}^{*}+\dot{J}_{2} \dot{q}\right] \\
& +M \hat{N}_{2} N_{1} \gamma .
\end{aligned}
$$

This control law has the same closed-loop impedance as does Equation 18 in the special case that $\Omega_{1}=\Lambda_{1}$ and $\Omega_{2}=\Lambda_{2}$. Since it does not require Cartesian force sensors to be mounted at the first- and second-priority PORs, this control law will be referred to as the "zero-force" dual-priority control law. (The forces are not zero, but we do not need to measure them.)

\section{B. Analysis}

The analysis of the zero-force dual-priority Cartesian impedance control law (Equation 26) is similar to the analysis performed in Section III-D. Substituting Equation 26 into the equation of motion (Equation 1) and using the fact that $\ddot{x}_{1}=J_{1} \ddot{q}+\dot{J}_{1} \dot{q}$, the dynamics of the first-priority POR are:

$$
\Lambda_{1} \ddot{x}_{1}+f_{1}^{*}=\Lambda_{1} J_{1} M^{-1} \tau \text {. }
$$

Note the similarities between the closed-loop impedance above and the desired impedance in Equation 2. When all external loads are applied to the first-priority POR such that $\tau=J_{1}^{T} f_{1}$, then:

$$
\Lambda_{1} J_{1} M^{-1} \tau=f_{1} .
$$

and Equation 27 is identical to Equation 2. When external loads are applied to both PORs such that $\tau=J_{1}^{T} f_{1}+J_{2}^{T} f_{2}$, then loads applied to the second-priority POR, $f_{2}$, are projected through $\Lambda_{1} J_{1} M^{-1} J_{2}^{T}$ onto the first-priority POR:

$$
\Lambda_{1} J_{1} M^{-1} \tau=f_{1}+\Lambda_{1} J_{1} M^{-1} J_{2}^{T} f_{2} .
$$

Substituting Equation 26 into the equation of motion (Equation 1) and using the fact that $\ddot{x}_{2}=J_{2} \ddot{q}+\dot{J}_{2} \dot{q}$, the dynamics of the first-priority POR are:

$$
\begin{aligned}
\ddot{x}_{2}= & J_{2} M^{-1} \tau-J_{2} \hat{N}_{2} J_{1}^{+}\left(\Lambda_{1}^{-1} f_{1}^{*}+\dot{J}_{1} \dot{q}\right) \\
& -J_{2} \hat{J}_{2}\left(\Lambda_{1}^{-1} f_{2}^{*}+\dot{J}_{2} \dot{q}\right)+\dot{J}_{2} \dot{q} \\
= & J_{2} M^{-1} \tau-\left(I-J_{2} \hat{J}_{2}\right) J_{1}^{+}\left(\Lambda_{1}^{-1} f_{1}^{*}+\dot{J}_{1} \dot{q}\right) \\
& -J_{2} \hat{J}_{2} \Lambda_{1}^{-1} f_{2}^{*}+\left(I-J_{2} \hat{J}_{2}\right) \dot{J}_{2} \dot{q} .
\end{aligned}
$$

Multiplying the above by $J_{2} \hat{J}_{2}$, the resulting closed-loop behavior at the second-priority POR is:

$$
J_{2} \hat{J}_{2} \ddot{x}_{2}=J_{2} \hat{J}_{2}\left(J_{2} M^{-1} \tau-\Lambda_{2}^{-1} f_{2}^{*}\right) .
$$

If all external loads are applied to the second-priority POR, then $\tau=J_{2}^{T} f_{2}$ and Equation 28 becomes Equation 21 for $\Omega_{2}=\Lambda_{2}$. If loads are applied to both PORs, $\tau=J_{1}^{T} f_{2}+J_{2}^{T} f_{2}$, then the load applied to the first-priority POR is projected onto the second-priority POR.

\section{Simulation}

The dual-priority approach proposed in this paper is compared to both a single-priority approach and the Sentis-Khatib dual-priority control law (Section III-E) in the context of a simple regrasp problem.

\section{A. Overview}

Figure 2 illustrates the simulation scenario. Both fingers are intially in contact with an object and touching it lightly (the entire manipulator is illustrated in Figure 1). The control problem is to move one finger while the other finger applies a small inward force resulting from a constant impedance. For example, this behavior could be important in an assembly application where one finger needs to maintain a constant force (perhaps in order to keep a part in place) while the other finger moves into a new grip position. What makes this a potentially difficult problem is that the first finger is not fully mobile with respect to the second finger. In view of Figure 1, notice that the two fingertips may only move independently in a plane; the wrist must swivel in order to move one of the fingers out of plane. It is not possible to move one finger to an arbitrary position while keeping both the position and orientation of the second finger fixed. If the position reference of the first finger cannot be fully reached while maintaining the pose and impedance of the second finger, then one or the other objective 


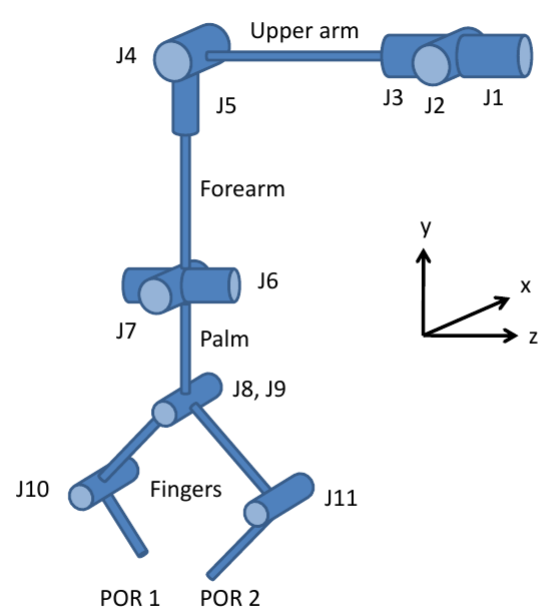

Fig. 1. The bifurcated manipulator (not drawn to scale) used in the simulations. A POR is defined at the end of each "finger."

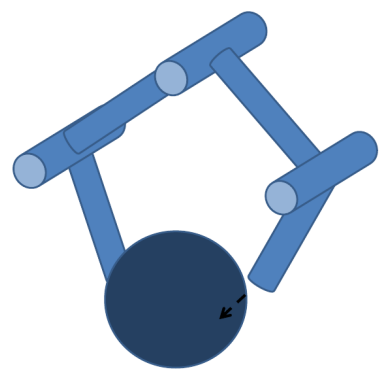

Fig. 2. The manipulation scenario. The two-finger manipulator is initially contacting the object lightly. At the start of the simulation, the right finger impedance position reference changes so that the finger will move in the direction of the dashed arrow. The left finger impedance remains unchanged so that it will continue to apply a light inward force on the object. The dual-priority control law must move the right finger without changing the impedance of the left finger.

must be "sacrificed." In this application scenario, it is desirable to sacrifice those aspects of the first finger objective so that regardless of how the finger is commanded to move, the second finger is assured of maintaining a constant impedance.

The entire manipulator is illustrated in Figure 1. The three links proximal to the bifurcation will be referred to, in order proximal to distal, as: the "upper arm", the "forearm", and the "palm." The two branches will be referred to as the "fingers." The lengths, masses, and moments of inertia are given in Table I. Note that the finger masses and inertias are large enough to be of the same order as the arm. A POR is defined at the tip of each of the two fingers.

\section{B. Comparison with a single-priority control law}

For reference, the dual-priority control law proposed in this paper was compared to a single priority control law. Compared with the dual-priority control law, this is a simpler alternative and should only be discarded when significant advantages are offered by a dual-priority law.

1) Single-priority control law: The single-priority law is:

$$
u=J^{T} f^{*}+M J^{+} \dot{J} \dot{q}+M N B_{j} \dot{q} .
$$

This achieves a desired impedance,

$$
\Lambda_{s} \ddot{x}_{s}+f_{s}^{*}=f_{s},
$$

where $\ddot{x}_{s}^{T}=\left(\ddot{p}_{1}, \dot{\omega}_{1}, \ddot{p}_{2}\right)$ is a generalized acceleration, $\Lambda_{s}$ is the passive manipulator inertia projected into the corresponding operational space coordinates, and $f_{s}$ is the generalized force. Both desired impedances are combined into a single impedance objective:

$$
f_{s}^{*}=\left(\begin{array}{ccc}
B_{1}^{p} & \mathbf{0} & \mathbf{0} \\
\mathbf{0} & B_{1}^{r} & \mathbf{0} \\
\mathbf{0} & \mathbf{0} & B_{2}^{p}
\end{array}\right) \dot{x}_{s}+\left(\begin{array}{ccc}
K_{1}^{p} & \mathbf{0} & \mathbf{0} \\
\mathbf{0} & K_{1}^{r} & \mathbf{0} \\
\mathbf{0} & \mathbf{0} & K_{2}^{p}
\end{array}\right) \tilde{x}_{s}
$$

where $K_{1}^{p}=\operatorname{diag}(45)$ (Newtons per Meter) and $B_{1}^{p}=$ $\operatorname{diag}(25)$ (Newton-seconds per Meter) are the desired translational stiffness and damping of POR $1, K_{1}^{r}=\operatorname{diag}(10)$ and $B_{1}^{r}=\operatorname{diag}(5)$ are the desired angular stiffness and damping of POR 1 , and $K_{2}^{p}=\operatorname{diag}(20)$ and $B_{2}^{p}=\operatorname{diag}(25)$ are the desired translational stiffness and damping of POR 2. The corresponding Jacobian is

$$
J=\left(\begin{array}{c}
J_{1}^{p} \\
J_{1}^{r} \\
J_{2}^{p}
\end{array}\right)
$$

where $J_{1}^{p}$ and $J_{1}^{r}$ describe the Cartesian translational and angular velocities (respectively) at POR 1 and $J_{2}^{p}$ is the translational Jacobian for POR 2.

2) Dual-priority control law: The single-priority control law above was compared to the dual priority control law of Equation 18. The first priority objective was to realize a translational and angular desired impedance at POR 1,

$$
\Lambda_{1} \ddot{x}_{1}+f_{1}^{*}=f_{1} .
$$

$\ddot{x}_{1}=\left(\ddot{p}_{1}, \dot{\omega}_{1}\right)$ is the acceleration in $S E(3) . \Lambda_{1}$ is the corresponding passive operational space inertia, $f_{1}^{*}$ is the desired damping and stiffness,

$$
f_{1}^{*}=\left(\begin{array}{cc}
B_{1}^{p} & \mathbf{0} \\
\mathbf{0} & B_{1}^{r}
\end{array}\right) \dot{x}_{1}+\left(\begin{array}{cc}
K_{1}^{p} & \mathbf{0} \\
\mathbf{0} & K_{1}^{r}
\end{array}\right) \tilde{x}_{1}
$$

and $f_{1}$ is the corresponding externally applied load. The second priority objective was to realize just a translational impedance at POR 2 - not an angular impedance,

$$
\Lambda_{2} \ddot{p}_{2}+f_{2}^{*}=f_{2},
$$

where $\Lambda_{2}$ is the passive manipulator inertia projected into the translational Euclidean coordinates for POR 2, and $f_{2}$ is the externally applied force at POR 2 . The desired POR 2 damping and stiffness is:

$$
f_{2}^{*}=B_{2}^{p} \dot{p}_{2}+K_{2}^{p} \tilde{p}_{2} .
$$

Figure 3 compares the performance of the two control laws when the first-priority POR reference pose is the same as 


\begin{tabular}{|c|c|c|c|c|c|}
\hline Link & Length & Mass & $I_{x x}$ & $I_{y y}$ & $I_{z z}$ \\
\hline upper arm & $0.38 \mathrm{~m}$ & $6.8 \mathrm{Kg}$ & 0.082 & 0.082 & 0.0082 \\
forearm & $0.35 \mathrm{~m}$ & $5.5 \mathrm{Kg}$ & 0.056 & 0.056 & 0.0056 \\
palm & $0.1 \mathrm{~m}$ & $2 \mathrm{Kg}$ & 0.01 & 0.01 & 0.001 \\
finger link & $0.1 \mathrm{~m}$ & $1 \mathrm{Kg}$ & 0.005 & 0.005 & 0.0005 \\
\hline
\end{tabular}

TABLE I

DYNAMIC PARAMETERS OF SIMULATED ARM. $I_{z} z$ IS THE MOMENT OF INERTIA ABOUT THE LINK AXIS. $I_{x} x$ AND $I_{y y}$ ARE THE REMAINING TWO ORTHOGONAL MOMENTS OF INERTIA. ALL FINGER LINKS HAVE THE SAME DYNAMIC PARAMETERS.

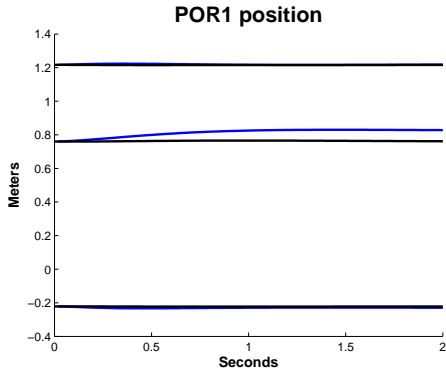

(a)

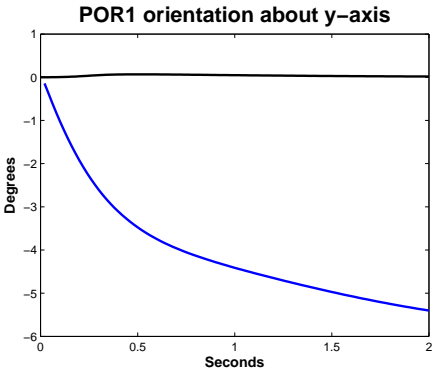

(b)

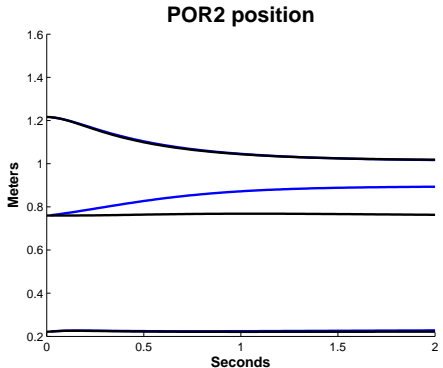

(c)

Fig. 3. Comparison between the dual-priority control law proposed in this paper (the solid black line) and a single-priority controller (the shaded blue line).

in the starting configuration, $p_{2}^{*}=p_{2}(0)$ and $r_{2}^{*}=r_{2}(0)$, the second-priority POR reference position is changed, $p_{2}^{*}=$ $(0.96,1.016,0)^{T}$, and no external forces are applied to the manipulator. First, notice that the dual-priority control law (the solid black line in Figure 3) keeps POR 1 in approximately the same position and orientation during the interaction while the single-priority control law (the shaded blue line) allows POR 1 to move. This is not suprising because the single priority control law does not enforce the prioritization. Second, notice that Figure 3(c) shows that the single-priority control law gets closer to achieving the POR 2 objective than the dual-priority controller. Since the single-priority controller allowed POR 1 to move and rotate from the reference, the $x$ coordinate of the POR 2 position (the glue line in Figure 3(c)) moves toward the 0.96 reference (although it does not reach the reference because it is balancing the POR 1 objective as well). Nevertheless, since the POR $2 y$-dimension (the top line in Figure 3(c)) is unconstrained with respect to the POR 1 objectives, both the single- and dual-priority control laws realize the $y$-dimension objective with approximately the same performance. In contrast, the dual-priority control law maintains constant POR 1 position and orientation. POR 2 position error is minimized in the directions that do not change POR 1 orientation and ignored in the directions that do.

\section{Comparison with Sentis-Khatib}

Section III-E demonstrated that the proposed dual-priority control law has certain theoretical advantages relative to the Sentis-Khatib control law. These differences have an important effect on the overall performance of the control law. The two control laws are compared under the same conditions used above to compare with the single priority law. The simulation compared the Sentis-Khatib control law in Equation 22 with the proposed dual-priority control law in Equation 18. Both control laws are parameterized with the desired first- and second-priority impedances given in Equations 30 and 31.

Figure 4(a) and (b) demonstrates that both dual-priority control laws realize the first-priority impedance almost equally well (the proposed control law does a bit worse in keeping the orientation constraint.) This is expected since it is possible to substitute either control law into the manipulator dynamics (Equation 1), solve for the POR 1 accelerations, and arrive at Equation 2. However Figure 4(c) illustrates the differences regarding POR 2. The control law of this paper (the solid black line) converges to the position nearest (Euclidian norm) the POR 2 reference position while the Sentis-Khatib control law converges elsewhere (the shaded blue line). This result demonstrates that the theoretically suboptimal performance demonstrated in Equation 24 can have a significant practical impact.

\section{CONCLUSION}

Multi-priority Cartesian impedance control is important because it enables the robot programmer to control a complex manipulator in terms of desired Cartesian impedances at multiple PORs. This is the natural extension of single endeffector control to branching manipulators. The prioritization is necessary to resolve conflicts between different objectives. Although multi-priority motion control can achieve prioritized motions that are similar to what can be achieved with multipriority impedance control, these motions are not robust and predictable when the possibility of contact exists. Manipulation and assembly applications, in particular, require exactly the type of behavior that this paper has considered: motion 


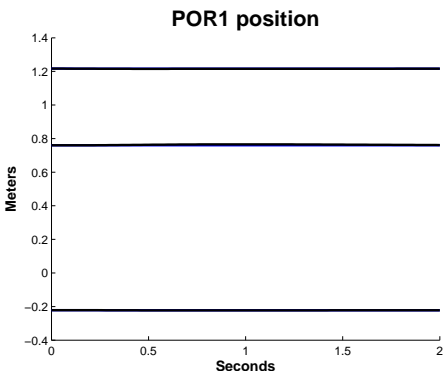

(a)

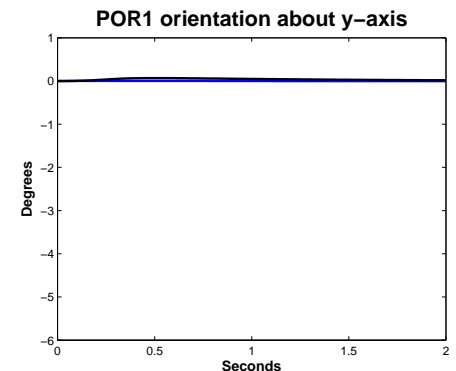

(b)

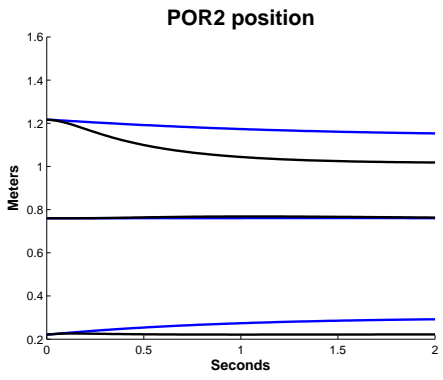

(c)

Fig. 4. Comparison between the dual-priority controller proposed in this paper (the solid black line) and the Sentis Khatib dual-priority controller (the shaded line). (a) and (b) show similar performance for both control laws with respect to the first priority impedance objective while (c) shows that the proposed controller has better performance with respect to the second priority impedance.

of a second-priority POR while the first-priority POR acts with a constant desired force or impedance. This paper makes two main contributions. First, we propose a locally optimal dual-priority impedance control law for the general case where end-effector load measurements may or may not be available. Second, we analyze the performance of the control law in theory and relative to the existing multi-priority impedance control literature.

\section{REFERENCES}

[1] B. T. Inc., "Products - WAM Arm," http://www.barrett.com/robot/products-arm.htm.

[2] A. Albu-Schaffer, S. Haddadin, C. Ott, A. Stemmer, T. Wimbock, and G. Hirzinger, "The DLR lightweight robot lightweight design and soft robotics control concepts for robots in human environments," Industrial Robot Journal, vol. 34, pp. 376-385, 2007.

[3] A. Liegois, "Automatic supervisory control of the configuration and behavior of multibody mechanisms," IEEE Trans. Syst. Man Cybernetics, pp. 868-871, 1977.

[4] Y. Nakamura, Advanced Robotics Redundancy and Optimization. Addison-Wesley, 1991.

[5] L. Sciavicco and B. Siciliano, "A solution algorithm to the inverse kinematic problem for redundant manipulators," IEEE Journal of Robotics and Automation, vol. 4, no. 4, 1988.

[6] L. Sentis and O. Khatib, "Synthesis of whole-body behaviors through hierarchical control of behavioral primitives," International Journal of Humanoid Robotics, 2005.

[7] R. Platt, O. Brock, A. H. Fagg, D. Karupiah, M. Rosenstein, J. Coelho, M. Huber, J. Piater, D. Wheeler, and R. Grupen, "A framework for humanoid control and intelligence," in Proceedings of the 2003 IEEE International Conference on Humanoid Robots, October 2003.

[8] P. Chiacchio, S. Chiaverini, L. Sciavicco, and B. Siciliano, "Closed-loop inverse kinematics schemes for constrained redundant manipulators with task space augmentation and task priority strategy," The International Journal of Robotics Research, vol. 10, no. 4, pp. 410-425, 1991.

[9] S. Chiaverini, "Singularity-robust task-priority redundancy resolution for real-time kinematic control of robot manipulators," IEEE Transactions on Robotics and Automation, vol. 13, no. 3, pp. 398-410, 1997.

[10] G. Antonelli, "Stability analysis for prioritized closed-loop inverse kinematic algorithms for redundant robotic systems," IEEE Transactions on Robotics, vol. 25, no. 5, pp. 985-994, 2009.

[11] M. Huber, "A hybrid architecture for adaptive robot control," Ph.D. dissertation, U. Massachusetts, 2000.

[12] N. Mansard and F. Chaumette, "Task sequencing for sensor-based control," IEEE Transactions on Robotics, vol. 23 , no. 1, pp. 60-72, 2007.

[13] N. Hogan, "Impedance control - an approach to manipulation: theory," Journal of dynamic systems measurement and control, vol. 107, pp. 1-7, 1985.
[14] C. Natale, B. Siciliano, and L. Villani, "Spatial impedance control of redundant manipulators," in IEEE Int'l Conf. on Robotics and Automation, 1999, pp. 1788-1793.

[15] A. Albu-Schffer and G. Hirzinger, "Cartesian impedance control techniques for torque controlled light-weight robots," in IEEE Int'l Conf. on Robotics and Automation, 2002, pp. 657-663.

[16] Y. Oh, W. Chung, and Y. Youm, "Extended impedance control of redundant manipulators based on weighted decomposition of joint space," Journal of Robotic Systems, vol. 15, no. 5, pp. 231-258, 1998.

[17] C. Ott, A. Kugi, and Y. Nakamura, "Resolving the problem of nonintegrability of nullspace velocities for compliance control of redundant manipulators by using semi-definite lyapunov functions," in IEEE Int'l Conf. on Robotics and Automation, 2008, pp. 1999 - 2004.

[18] L. Sentis, "Synthesis and control of whole-body behaviors in humanoid systems," Ph.D. dissertation, Stanford University, July 2007.

[19] M. Spong, S. Hutchinson, and M. Vidyasagar, Robot Modeling and Control. John Wiley and Sons, Inc., 2005.

[20] R. Murray, Z. Li, and S. Sastry, A Mathematical Introduction to Robotic Manipulation. CRC Press, 1994. 